\title{
Modélisation numérique d'un écoulement permanent dans un réseau hydraulique maillé à surface libre, en régime fluvial
}

\author{
Numerical modelling of a steady state flow \\ in a free surface looped hydraulic network \\ under fluvial conditions
}

\author{
Jean-Pierre Baume et Michel Poirson
}

Ingénieurs au CEMAGREF d'Antony

Le calcul de la ligne d'eau dans un réseau maillé à surface libre est un problème fréquemment rencontré en aménagement de rivière, en particulier dans les zones de marais ou pour l'étude du comportement des biefs usiniers.

Dans le maillage, on ignore la répartition des débits entre les différentes branches. Le calcul peut se faire en utilisant la méthode itérative de Hardy-Cross, mais la convergence est lente, car les pertes de charge linéaires dépendent à la fois du débit et de la ligne d'eau. On peut aussi utiliser un modèle en régime transitoire avec des conditions constantes aux limites, mais celui-ci réclame une ligne d'eau initiale et se stabilisera d'autant moins vite qu'on était éloigné au départ de la solution.

Nous présentons une nouvelle méthode itérative, à convergence rapide, basée sur la résolution d'un système d'équations linéarisées. Celles-ci sont classées de manière à permettre une décomposition en blocs de la matrice creuse du système initial qui peut alors être transformé pour simplifier la recherche de la solution.

Cette méthode a été programmée sur ordinateur et une application de ce modèle à un cas réel est jointe à titre d'exemple.

\section{Description du réseau hydraulique}

On suppose connu le sens d'écoulement. Le réseau hydraulique est décomposé en tronçons homogènes : les biefs définis entre un nœud amont et un nœud aval.

On considère l'ensemble des biefs dans lesquels on ne connaît pas a priori le débit. Si cet ensemble n'est pas connexe on y inclut d'autres biefs du réseau (où le débit est connu) pour obtenir un ensemble connexe. C'est cet ensemble que l'on nomme maille. Ainsi le réseau ne contient qu'une seule maille aussi complexe que l'on veut. On distinguera les biefs appartenant à la maille et ceux qui sont en dehors.
La maille commence toujours par un défluent. Si le modèle a plusieurs conditions aval la maille ne se referme pas. Sinon elle se termine par un confluent et peut alors être suivie par d'autres biefs n'appartenant pas à la maille.

On appellera nœud aval de maille, un nœud de la maille d'où il ne part aucun bief de maille.

\section{Modélisation d'un bief}

La géométrie d'un bief est déterminée par les profils en travers caractéristiques de la forme et du volume du cours d'eau. A partir de ces sections de donnée, des sections de calcul plus rapprochées sont interpolées pour augmenter la précision de la résolution numérique.

\section{Hypothèses et notations}

On considère que le lit de la rivière se compose d'un lit mineur et d'un lit moyen (voir fig. 1). L'écoulement est filaire (la surface libre est horizontale dans un profil en

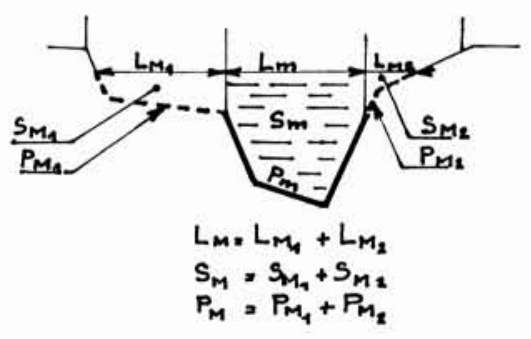

Figure 1. - Profil en travers du lit. 


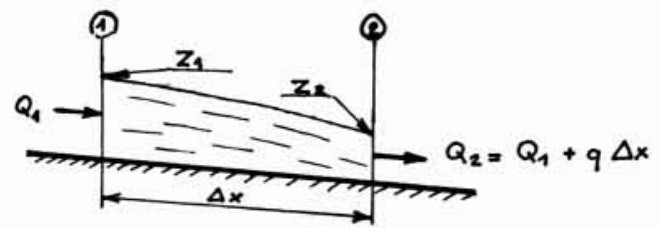

Figure 2 - Discrétisation de la ligne d'eau.

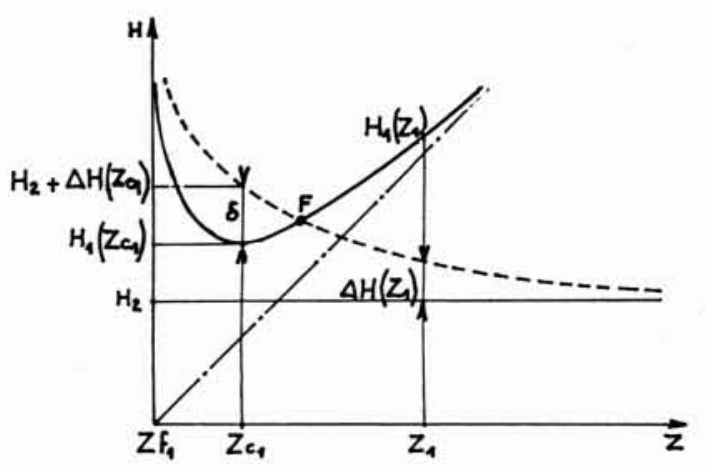

Figure 3 - Existence d'une solution pour la ligne d'eau. travers et les vitesses transversales sont négligeables). La répartition de débit entre les deux lits est donnée par la formulation DEBORD (LNH Chatou).

L'indice $m$ correspond au lit mineur, $M$ au lit moyen. Les variables sans indice s'appliquent à la section totale.

$Z$ : cote de la surface libre

$L$ : largeur au miroir

$S$ : surface mouillée

$P$ : périmètre mouillé

$R$ : rayon hydraulique

$Q$ : débit

$V$ : vitesse

$J$ : pente de la ligne de charge

$D_{e}$ : débitance équivalente

$K$ : coefficient de Manning-Strickler

$I$ : pente du fond

$x$ : abscisse de positionnement de la section

$g$ : accélération de la pesanteur

$\beta$ : coefficient de quantité de mouvement

$$
\beta=\frac{\iint v^{2} d S}{V^{2} S}
$$

\section{Formulation DEBORD [4]}

$$
\begin{gathered}
J=Q^{2} / D_{e}^{2} \\
D_{e}=K_{m} S_{m} A R_{m}^{2 / 3}+K_{M} \sqrt{S_{M}^{2}+S_{m} S_{M}\left(1-A^{2}\right)} R_{M}^{2 / 3}
\end{gathered}
$$

où le coefficient $A$ représente la loi expérimentale de variation du débit mineur en raison de la présence du lit moyen.

$$
\begin{array}{ll}
A=A_{0}=0,9\left(\frac{K_{m}}{K_{M}}\right)^{-1 / 6} \quad \text { pour } & r=R_{m} / R_{M}>0,3 \\
A=\frac{1-A_{0}}{2} \cos \frac{\pi r}{3}+\frac{1-A_{0}}{2} & \text { pour } \quad 0 \leqslant r \leqslant 0,3
\end{array}
$$

Le coefficient de quantité de mouvement est estimé par :

$$
\beta=\left(\frac{\eta^{2}}{S_{m}}+\frac{1}{S_{M}}\right) \frac{S}{(1+\eta)^{2}}
$$

avec

$$
\eta=\frac{Q_{m}}{Q_{M}}=\frac{K_{m}}{K_{M}} \frac{S_{m} A}{\sqrt{S_{M}^{2}+S_{m} S_{M}\left(1-A^{2}\right)}}\left(\frac{R_{m}}{R_{M}}\right)^{2 / 3}
$$

répartition des débits dans les deux lits.
Le cas particulier $A=1$ donne la formulation classique des lits composés, les valeurs de $A$ différentes de 1 rendent compte de l'interaction entre les écoulements mineur et moyen.

\section{Equations}

Equation de continuité :

$$
\frac{\mathrm{d} Q}{\mathrm{~d} x}=q
$$

avec $q=0 \quad$ débit constant

$q>0 \quad$ apport du bassin versant

$q<0 \quad$ perte

( $q$ est indépendant de la ligne d'eau)

Equation dynamique :

$$
\frac{\mathrm{d} H}{\mathrm{~d} x}+\frac{V^{2}}{2 g} \frac{\mathrm{d} \beta}{\mathrm{d} x}+\frac{q V}{g S}(\beta-k)+J+J_{s}=0
$$

avec

$$
H=\beta \frac{\mathrm{V}^{2}}{2 g}+Z
$$

$k=0$ si $q \geqslant 0$ pas d'apport de quantité de mouvement $k=1$ si $q<0$ perte de quantité de mouvement $J_{s}=$ perte de charge unitaire à l'élargissement

\section{Calcul de la ligne d'eau [5]}

On connaît la condition amont en débit. En écoulement fluvial la ligne d'eau est intégrée pas à pas à partir de la cote aval.

Discrétisée entre deux sections (1) et (2) distante de $\Delta x$ (voir fig. 2), l'équation dynamique s'écrit :

$$
\begin{aligned}
H_{2}-H_{1}+ & \frac{\beta_{2}-\beta_{1}}{4 g}\left(V_{2}^{2}+V_{1}^{2}\right) \\
+ & \frac{q \Delta x}{2 g}\left(\left(\beta_{2}-k\right) \frac{V_{2}}{S_{2}}+\left(\beta_{1}-k\right) \frac{V_{1}}{S_{1}}\right) \\
& +\frac{J_{2}+J_{1}}{2} \Delta x+\xi \frac{\left(V_{1}-V_{2}\right)^{2}}{2 g}=0
\end{aligned}
$$

avec $\xi$ coefficient de perte de charge à l'élargissement (formule de Borda). 
On cherche la valeur $Z_{1}$ connaissant tous les paramètres en (2).

L'équation (1) peut se mettre sous la forme

$H_{1}=H_{2}+\Delta H\left(Z_{1}\right)$.

Il existe une solution si les courbes $H_{1}\left(Z_{1}\right)$ et $H_{2}+\Delta H\left(Z_{1}\right)$ se coupent.

Pour cela il faut que

$\delta=H_{2}+\Delta H\left(Z_{c 1}\right)-H_{1}\left(Z_{c 1}\right)>0$ (voir Fig. 3).

$Z_{c \text {, }}$ est la cote critique définie en (1) par :

$$
\beta_{1} \frac{Q_{1}^{2} L_{1}}{g S_{1}^{3}}=1
$$

$\delta>0$ : solution fluviale $F$

$\delta<0$ : il y a rupture de pente et passage $\operatorname{par} Z_{c 1}$ ou bien il $y$ a un ressaut.

\section{Remarque}

Le calcul de la ligne d'eau est fait pour un régime fluvial; néanmoins le modèle accepte les passages localisés en régime torrentiel et positionne le ressaut.

Les singularités (déversoirs, vannes) rencontrées dans les biefs sont prises en compte et font l'objet d'un traitement particulier.

Tri topologique appliqué à un réseau hydraulique [6]

Un écoulement fluvial étant régi par l'aval, le calcul d'une ligne d'eau se fait en remontant la rivière. Il faut donc trouver une relation donnant un ordre dans lequel on peut traiter les biefs à partir de la connaissance de la condition en cote aval. Un réseau hydraulique peut être représenté par un graphe orienté, connexe, acyclique sur lequel on effectue un parcours à l'aide de l'algorithme suivant :

On cherche un sommet sur lequel n'arrive aucun arc et d'où il ne part qu'un seul arc. On sélectionne, dans une liste, ce dernier puis on le supprime du graphe. On applique récursivement le procédé sur le sous-graphe obtenu tant qu'il existe des sommets ainsi définis.

Ensuite soit le problème est terminé, soit il reste des arcs non récupérés. Dans ce cas :

On cherche dans le sous-graphe restant un sommet sur lequel n'arrive aucun arc et d'où il part plus d'un arc. On sélectionne ces derniers puis on les supprime du graphe et on applique récursivement le procédé sur le sous-graphe obtenu tant qu'il existe des sommets ainsi définis.

Ensuite soit le problème est terminé soit il reste des arcs non récupérés. Dans ce cas on recommence sur le sousgraphe restant l'algorithme depuis le début.

En lisant à l'envers la liste des arcs ainsi obtenue on trouve une relation d'ordre partiel satisfaisante.

De la liste on tire également le repérage des biefs de maille. Ceux-ci sont groupés et commencent au premier défluent (les deux premiers biefs successifs qui ont le même nœud amont).

Si le modèle a plusieurs conditions en cote aval tous les biefs suivant le premier défluent dans la liste appartiennent à la maille. Si par contre la maille se referme, elle s'arrête au dernier confluent (le dernier bief qui a le même nœud aval qu'un autre bief situé dans la liste après le début de maille).

Calcul des biefs de maille [6]

\section{Solution initiale}

On fixe un débit initial dans chaque bief de maille en respectant la continuité des débits aux nœuds, en prenant par exemple une répartition au prorata de la section moyenne du bief (rapport du volume du bief aux cotes de débordement sur sa longueur).

Puis on calcule successivement la ligne d'eau dans chaque bief, en suivant l'ordre établi (voir au paragraphe précédent) et en prenant pour condition aval (dans le cas d'un nœud défluent) la moyenne des cotes obtenues par le calcul des biefs aval partant de ce nœud.

Soit une partie du bief $i$ définie par deux sections, on cherche la cote $Z_{j}^{j}$ dans la section $j$ amont, connaissant la cote $Z_{j+1}^{i}$ dans la section $j+1$ aval et le débit $Q_{j}^{i}$ s'écoulant dans la section $j$.

Cela revient à résoudre (numériquement) l'équation (1) qui peut se mettre sous la forme :

$$
f\left(Z_{j}^{i}, Z_{j+1}^{\prime}, Q_{j}^{\prime}\right)=0
$$

\section{Equations de correction}

La ligne d'eau corrigée sera solution de l'équation :

$$
f\left(Z_{j}^{i}+\Delta Z_{j}^{i}, Z_{j+1}^{i}+\Delta Z_{j+1}^{i}, Q_{j}^{i}+\Delta Q^{i}\right)=0
$$

En développant (3) en série de Taylor limitée aux termes du premier ordre : avec

$$
\text { (3) }-(2) \rightarrow a_{j}^{i} \Delta Z_{j}^{i}+b_{j}^{i} \Delta Z_{j}^{i}+c_{j}^{i} \Delta Q^{i}=0
$$

$$
a_{j}^{\prime}=\frac{\partial f}{\partial Z_{j}^{i}}, b_{j}^{\prime}=\frac{\partial f}{\partial Z_{j+1}^{i}}, c_{j}^{\prime}=\frac{\partial f}{\partial Q_{j}^{\prime}}
$$

On sait que $a_{j}^{\prime}$ n'est pas nul (la résolution de (2) est faite par la méthode de Newton où intervient le rapport $\left.f / a_{j}^{\prime}\right)$. On pose :

$$
\mathrm{d}_{j}^{i}=-\frac{\mathrm{b}_{j}^{i}}{a_{j}^{i}} \quad \text { et } \quad e_{j}^{i}=-\frac{c_{j}^{i}}{a_{j}^{i}}
$$

Pour un bief $i$ de $n$ sections, les $(n-1)$ équations (4) deviennent :

$$
\begin{gathered}
\Delta Z_{1}^{i}=\mathrm{d}_{1}^{i} \Delta Z_{2}^{i}+e_{1}^{i} \Delta Q^{\prime} \\
\overline{\Delta Z_{j}^{i}}=\overline{\mathrm{d}_{j}^{i} \Delta Z_{j+1}^{i}+e_{j}^{i} \Delta Q^{i}}- \\
\Delta \bar{Z}_{n-1}^{i}=-\mathrm{d}_{n-1}^{i} \Delta \bar{Z}_{n}^{i}+\bar{e}_{n-1}^{i} \Delta \bar{Q}^{i}
\end{gathered}
$$

En condensant ces équations relatives à deux sections, on obtient une relation caractéristique du bief :

$$
\Delta Z_{i}^{i}=\mathscr{O}^{i} \Delta Z_{n}^{i}+\mathcal{E}^{i} \Delta Q^{i}
$$

avec $i=$ indice du bief

$1=$ indice de la section amont du bief

$n=$ indice de la section aval du bief 
et

avec

$$
\begin{gathered}
\mathcal{Q}^{i}=\prod_{j=1}^{n} \mathrm{~d}_{j}^{i} \\
\mathcal{E}^{i}=\mathcal{O}^{i} \sum_{k=1}^{n} \frac{e_{k}^{i}}{\prod_{j=k}^{n} \mathrm{~d}_{j}^{i}}
\end{gathered}
$$

$$
\mathrm{d}_{n}^{i}=1 \quad \text { et } \quad e_{n}^{i}=0
$$

Les autres relations dont on dispose sont :

- pour tous les nœuds de la maille qui ne sont pas nœuds aval de maille :

- la continuité des débits

$$
\sum_{T}^{k} \varepsilon^{i} \Delta Q^{i}=0
$$

avec $\quad k=$ nombre de biefs de maille reliés au nœud $\varepsilon^{\prime}=1$ bief amont et $\varepsilon^{\prime}=-1$ bief aval

- l'égalité des cotes

$$
Z_{u}^{\prime}+\Delta Z_{u}^{i}=Z_{v}^{\prime}+\Delta Z_{v}^{\prime}
$$

soit $k-1$ relations

avec $u, v=\{1$ si bief partant du nœud

$$
\{\text { si bief arrivant sur le nœud }
$$

$i, \ell=$ biefs reliés au nœud

- pour tous les nœuds aval de maille :

$\Delta Z_{n}^{i}=0$ (8) si la condition aval est une cote donnée

ou $\Delta Z_{n}^{i}=g\left(\Delta Q^{i}\right)$ si courbe de tarage aval (dans ce cas un seul bief par nœud aval)

\section{Résolution du système linéaire des équations de correc- tion}

On a 3 inconnues par bief $i$,

en cote $=\Delta Z_{1}^{i}$ à l'amont

en cote $=\Delta Z_{n}^{i}$ à l'aval

en débit $=\Delta Q^{i}$ sur tout le bief

Soit $N$ le nombre de biefs de maille. On pourrait résoudre le système des $3 N$ équations. Mais il sera de toute façon nécessaire de refaire après correction des débits un calcul général de ligne d'eau pour obtenir la cote dans chacune des sections des biefs.

On voit donc que les seules inconnues qui nous intéressent directement sont les $\Delta Q^{i}$.

On écrit les équations dans l'ordre suivant :

- les relations de type (6)

puis des relations de type (7) ou (8), de manière à obtenir un groupe de $N$ équations au total;

- les $N$ relations de type (5)

- les $N$ relations restantes de type (7) ou (8)

On voit apparaître des blocs carrés de rang $N$

$$
\begin{aligned}
& \left|\begin{array}{lll}
A & B & C \\
D & E & F \\
G & H & I
\end{array}\right| \cdot\left|\begin{array}{c}
\Delta Q \\
\Delta Z_{1} \\
\Delta Z_{n}
\end{array}\right|=\left|\begin{array}{l}
C_{1} \\
C_{2} \\
C_{3}
\end{array}\right| \\
& \text { Matrice Vecteur Constante }
\end{aligned}
$$

soit $M . V=K$

en multipliant (9) par une matrice de passage $P$ convenable

$$
(P . M) \cdot V=P . K
$$

on obtient un système de $N$ équations en $\Delta Q$

$$
A_{0} \cdot \Delta Q=C_{0}
$$

avec

$$
\begin{aligned}
& A_{0}=A-B \cdot D+X_{0} \cdot(H \cdot D-G) \\
& C_{0}=C_{1}-X_{0} \cdot C_{3} \\
& X_{0}=(B . F-C) \cdot X_{1}^{-1} \\
& X_{1}=H . F-I
\end{aligned}
$$

La méthode n'est valable que si la matrice $X_{1}$ est inversible. Or, celle-ci présente la particularité de toujours pouvoir se mettre sous la forme d'une matrice bande bidiagonale supérieure dont tous les éléments de la diagonale principale sont non nuls, ce qui garantit la régularité de $X_{1}$.

On peut alors inverser $X_{1}$ rapidement par la méthode de Gauss - Jordan. Le système (10) est résolu par la méthode de Gauss avec un pivot partiel.

\section{Remarque}

Le passage du système (9) au système (10) présente des avantages au niveau de la programmation :

- il permet de réduire la place mémoire nécessaire au stockage des variables ainsi que le nombre d'opérations (donc le temps de calcul et la précision). L'ordre de grandeur est de 6 fois dans les deux cas;

- il met en évidence le rôle de la matrice $F$, lors de l'inversion de $X_{1}$.

$H$ et $I$ ne contiennent que des $0,1,-1$.

$F$ est la matrice diagonale qui contient les coefficients $\boldsymbol{C}^{i}$ des équations (5). Ceux-ci traduisent dans chaque bief de maille la dépendance de la cote amont par rapport à la cote aval. Cette dépendance peut devenir très faible dans les cas où les tirants d'eau sont proches de la hauteur normale sur une partie du bief ou s'il y a présence de singularités en régime dénoyé.

Ayant localisé les coefficients $\omega^{\prime}$ négligeables, on peut, en bloquant arbitrairement ceux-ci à une valeur minimum, pallier une cause d'imprécision numérique.

\section{Comment obtenir $X_{1}=H . F-I$ sous forme bande?}

Les solutions dépendent du choix des $N$ relations de type (7) ou (8) et du classement des composantes du vecteur $\Delta Q$. La matrice $F$ étant diagonale le problème est analogue à celui de mettre $X_{1}^{\prime}=H-I$ sous forme bande. $X_{1}^{\prime}$ caractérise les relations entre les variations de cotes des biefs de maille reliés au même nœud, $H$ correspond aux cotes amont et $I$ aux cotes aval.

Pour chacun des nœuds de maille qui ne sont pas nœuds aval de maille (voir définitions au paragraphe " Description du réseau hydraulique "), on construit la liste des biefs de maille qui sont reliés à ce nœud.

Sur l'ensemble de ces listes certains biefs vont n'apparaitre qu'une seule fois, ce sont les biefs aval de maille.

On sélectionne une liste $\ell_{1}$ contenant au moins un bief aval de maille.

On met en tête de $\ell_{1}$ un bief aval de maille et en queue un bief $i$ (s'il existe) appartenant à une autre liste $\ell_{2}$. On place en tête de celle-ci le bief $i$ et en queue un bief (s'il existe) appartenant aussi à une liste différente de $\ell_{1}$ et $\ell_{2}$. On élimine de $\ell_{2}$ les biefs communs avec $\ell_{1}$ puis on concatène $\ell_{1}$ et $\ell_{2}$. On continue le processus jusqu'à l'obtention d'une liste unique. $\mathrm{Si}$ à un moment donné il n'existe pas de bief en queue de liste pour assurer le 


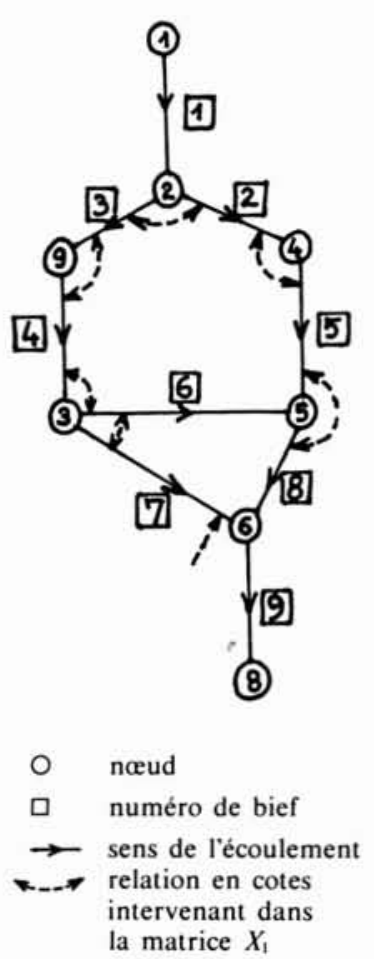

Figure 4 - L’Aisne à Sainte-Ménéhould.

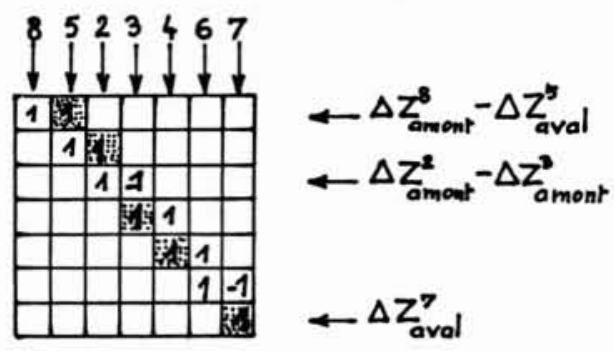

Figure 5 - Matrice $X^{\prime}$, bidiagonale.

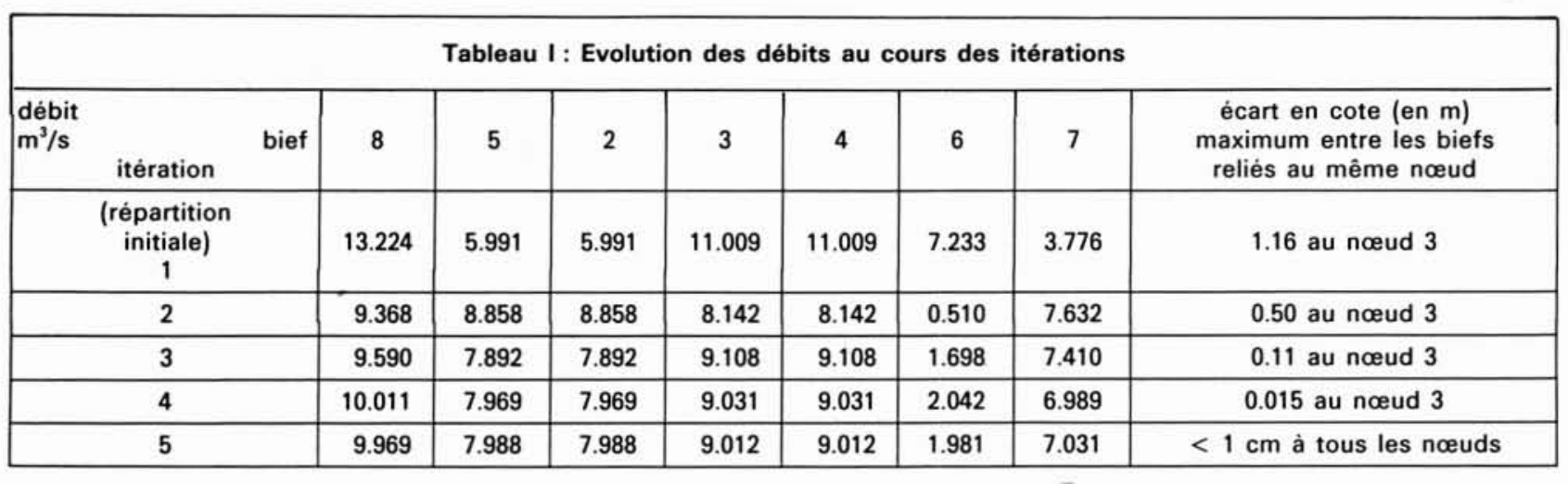

chaînage celui-ci se fait avec une liste contenant un bief aval de maille.

La liste finale correspond à l'inverse du classement des composantes du vecteur $\Delta Q$ et permet le choix des relations de type (7) et (8) constituant les blocs $G, H, I$.

Application

\section{Programmation}

Le modèle complet comprend deux programmes écrits en langage FORTRAN. Le premier appelé TALWEG traite la géométrie transformant les sections de données en sections de calcul, effectue le tri topologique, et donne matrices. Le calcul hydraulique se fait dans FLUVIA: calcul de ligne d'eau initiale, corrections des débits, puis itérations jusqu'à l'égalité des cotes aux næuds avec une précision inférieure ou égale à $1 \mathrm{~cm}$.

Ce résultat est obtenu en moyenne au bout de 5 itérations. Le calcul ne converge pas (en pratique arrêt à la $10^{\circ}$ itération) si le sens d'écoulement d'un bief a été mal choisi.

\section{Exemple}

Modélisation d'un cas réel : la rivière l'Aisne à SainteMénéhould.

En entrée des données les deux nœuds amont et aval suffisent pour repérer un bief. Le programme lui affecte ensuite un numéro correspondant à l'ordre dans lequel ce bief a été introduit en donnée (voir fig. 4).

Le tri topologique donne une succession logique des numéros de biefs permettant le calcul de la ligne d'eau depuis l'aval :

$$
9,8,7,6,4,5,3,2,1
$$

On sélectionne ensuite les biefs appartenant à la maille :

$$
8,7,6,4,5,3,2
$$

Puis on tire l'ordre de remplissage du vecteur $\Delta Q$ :

$$
8,5,2,3,4,6,7
$$

La matrice $X_{1}^{\prime}$ est bidiagonale (voir fig. 5. Les termes en grisé correspondent aux variations de cotes aval, les autres termes se rapportent aux variations de cotes amont).

En introduisant un débit $\left(17 \mathrm{~m}^{3} / \mathrm{s}\right)$ au næud (1), une cote aval 133,86 NGF au næud (8) et un strickler uniforme de 25 l'essai converge en 5 itérations (voir tableau I). tous les renseignements nécessaires au remplissage des 


\section{Conclusion}

La méthode itérative présentée est un outil efficace pour le calcul des réseaux maillés complexes en écoulement permanent à surface libre.

Le système linéarisé formé par les équations des écoulements dans une maille est réduit à un système fournissant seulement les débits.

Cette réduction est rendue possible par la décomposition en blocs de la matrice du système initial et par un classement rigoureux des biefs. La résolution du système est plus simple réduisant ainsi le temps de calcul et la place mémoire nécessaire au programme.

Le modèle a été appliqué avec succès à plusieurs cas réels de complexité moyenne (15 biefs). La convergence a été rapide même avec une répartition initiale des débits éloignée de la solution.

\section{Bibliographie}

[1] VREUGDENHIL C.B. - Computational methods for channel flow, committee for hydrological research TNO. Proceedings of technical meeting 26, 1973.

[2] DEMIDOVITCH et MARON. - Eléments de calcul numérique. Editions MIR, 1973.

[3] BARANGER J. - Introduction à l'analyse numérique. Editions Hermann, 1977.
[4] NICOLET G., UAN M. - Ecoulements permanents à surface libre en lits composés. La Houille Blanche - n 1, 1979.

[5] POCHAT R. - Ecole d'été de mécanique des fluides, hydraulique à surface libre. Hanoï, 1980.

[6] POIRSON M. - Solution originale pour résoudre les systèmes maillés complexes. Note interne au CEMAGREF, 1981. 